\section{Glosse}

\section{Darmbakterien zur Stimmungsaufhellung}

— Die Quelle des Glücks liegt im Darm. Auch wenn Kollege Eckart von Hirschhausen behauptet, einmal am Tag kräftig gelacht sei für die Gesundheit wichtiger als einmal richtig gekackt, dürfte doch die Kombination von beidem am segensreichsten sein. Auch für Voltaire waren die wichtigsten Indikatoren für ein glückliches Dasein: ein gut gefülltes Portemonnaie, eine hervorragende Köchin und eine gute Verdauung. Und vom alten Fritz stammt ein Satz, mit dem er der Gastroenterologie sogar den Vorrang vor der Philosophie eingeräumt hat: Es ist wichtiger, dass der Mensch gut verdaut, als dass er um die letzten Dinge dieser Welt Bescheid weiß.

Dass bei der Entfaltung solcher Glücksgefühle Darmbakterien entscheidend beteiligt sein könnten, scheint zunächst kaum vermittelbar, zumal wir diese ja bisher vorwiegend als Krankheitsverursacher und weniger als Glücksbringer wahrgenommen haben. Denken Sie an Ihre letzte Reisediarrhö - die hat Sie doch

Unglück aus dem Bauch? bestimmt nicht gerade glücklich gemacht, oder? Eine wissenschaftliche Erklärung für einen Kausalzusammenhang zwischen Darm und Glück könnte ein Experiment liefern, das jetzt bei Mäusen durchgeführt wurde. Sie erhielten randomisiert entweder eine normale oder eine mit Lactobacillus-rhamnosus-Bakterien versetzte Brühe zum Fressen. Und siehe da, die probiotischen Bakterien entfalteten bei den Tieren eine günstige Wirkung auf ängstliches und depressives Verhalten. Die Forscher vom University College Cork glauben, dass die Bakterien die Hirnchemie verändern und über die Stressachse die Ausschüttung von Stresshormonen verringern. Ob Bakterien wirklich eine Alternative zu Psychopharmaka sein können, bleibt weiter abzuklären.

Dr. med. Peter Stiefelhagen -

HYPONATRIÄMIE-GEFAHR

\title{
Auch Läufer sollen nicht über den Durst trinken
}

- Nach den Empfehlungen amerikanischer Getränkehersteller sollen Athleten $1200 \mathrm{ml}$ Flüssigkeit pro Stunde aufnehmen. Schon $2 \%$ Dehydratation würden sich negativ auf die Leistung auswirken. Unter diesem Druck versuchen Ausdauersportler, so viel Flüssigkeit wie nur möglich in sich hineinzupressen. Doch viele sind sich der drohenden Folgen nicht bewusst, wie eine US-Studie mit 197 Läufern zeigt.

Fast die Hälfte der Sportler tranken während des Laufs "nach Plan", d. h., auch wenn sie nicht durstig waren. Nur ein Drittel der Athleten wusste, dass zu viel Flüssigkeit die Gefahr einer Hyponatriämie („,exercise-associated hyponatremia", EAH, Salzverlust bei Ausdauerleistung) mit sich bringt. Die Folge können neurologische Beschwerden sein, die schlimmstenfalls zum Tod führen. Frühere Untersuchungen zeigen, dass bis zu 13\% der Marathonläufer mit einer Hyponatriämie durch die Zielgerade laufen. ST .

J Sports Med 2011; 45: 646-649

\section{SIMPLER TEST FÜR DIE PRAXIS}

\section{Schon leichte kognitive Einbußen sind lebensgefährlich}

- Ältere Menschen mit geistigen Leistungseinbußen haben eine verkürzte Lebenserwartung. Das gilt bereits bei leichten Formen kognitiver Einschränkung. Das Team um Greg Sachs von der Indiana University School of Medicine in Indianapolis hatte 3957 Personen im Alter zwischen 60 und 102 Jahren für ihre Studie rekrutiert. Die Patienten waren in den Jahren 1991 bis 1993 einem Kognitionstest mit dem sog. "Short Portable Mental Status Questionnaire" unterzogen. Je nach dem Testergebnis wurden sie drei Gruppen zugeordnet: keine (3157 Probanden), leichte (533) oder mäßige bis schwere geistige Einschränkungen (267). Im Jahr 2006 wurden mithilfe des National Death Index die Überlebenskurven berechnet.

Im Nachbeobachtungszeitraum waren $60,3 \%$ aller Studienteilnehmer verstorben: $57,4 \%$ aus der Gruppe ohne kognitive Einschränkungen, $68,1 \%$ aus der Gruppe mit leichten und $78,7 \%$ aus der Gruppe mit schweren Einbußen. Sowohl leichte als auch mäßige bis schwere geistige Defizite waren mit erhöhter Sterblichkeit assoziiert, und zwar unabhängig von anderen Risiken (Hazard Ratio [HR] 1,2 für leichte, HR 1,5 für mäßige bis schwere Defizite).

RB .

Sachs GA et al. Ann Intern Med 2011; 155:300-8

\section{SPMSQ}

Das "Short Portable Mental Status Questionnaire" ist ein simpler, zehn Punkte umfassender Test, der jederzeit unter Praxisbedingungen anwendbar ist (nach Pfeiffer E. A short portable mental status questionnaire for the assessment of organic brain deficit in elderly patients. J Am Geriatr Soc 1975; 23: 433-41; Testformular und Auswertung einzusehen zum Beispiel auf http:// www.npcrc.org/usr_doc/adhoc/ psychosocial/SPMSQ.pdf) 